\title{
LES CONTES D'AMOUR DANS LES MILLE ET UNE NUITS: DE QUELQUES CARACTERISTIQUES
}

Por

EDGARD WEBER

Lorsqu'on évoque les Mille et Une Nuits on pense trè̀s souvent à des amours interdits, à des scènes érotiques que la morale officielle désavoue... bref, à une manière d'être hors de la norme que le désir ou le phantasme ne cessent d'investir. Mais à regarder de plus près ces contes séculaires, il s'avère que la part proprement érotique est bien moins importante que ce qu'on imagine généralement. Certes, l'amour est présent, mais il n'est pas uniforme. Et si le conte se termine généralement par le mariage du héros et de l'héroïne, à savoir par le retour à la norme, ceux-ci peuvent être des princes mais aussi des fils de marchands ou de simples manants que la société a tout intérêt à voir unis. Ce mariage final n'est donc pas une pure convention, pour simplement bien terminer l'histoire. Sa permanence même est un signe qui mérite d'être analysé. Il répond pour ainsi dire à une structure globale du conte si l'on accorde au texte quelque importance ainsi qu'à la symbolique qui précisément l'anime en profondeur.

L'importance du mariage

Ce mariage est la réponse nécessaire à une situation initiale particulièrement négative, dans laquelle le héros, essentiellement entouré d'hommes, est éloigné de tout principe féminin. Son mariage ne peut être conçu alors que dans le sens d'une heureuse rencontre du principe masculin avec le principe féminin, sans laquelle, dans un premier temps, il n'y aurait jamais d'histoire à raconter. Ce point mérite d'être explicité quleque peu.

Le conte débute généralement avec l'absence totale de femmes et l'évocation d'un vieux roi sans enfants. Cette situation initiale où 
prédomine le principe masculin est vite génératrice de violence ${ }^{1}$ tant que le principe féminin n'y est pas rapidement intégré. Marie-Louise von Franz explique la valeur symbolique du roi : "Le roi incarne un principe divin dont dépend entièrement le bien-être psychique et physique du pays" 2 . Or, tant que ce principe est solide, fécond et jeune, la vie du peuple tout entier n'a rien à craindre. Mais si le roi venait à vieillir sans descendant, la vie-même du peuple serait compromise et exposée à sa disparition.

Le conte illustre donc la rencontre harmonieuse entre le principe masculin et le principe féminin, sans laquelle, symboliquement, le peuple ne pourrait vivre. Le mariage du prince ou de la princesse est la garantie de survie du peuple. C'est pour cette raison que le conte doit en principe se terminer avec le mariage légal et reconnu. Toute infraction à cette règle qui reflète en fait un ordre cosmique, doit être punie et rectifiée. Il est donc tout à fait naturel que les fauteurs de trouble soient châtiés, mais le conte aura eu le temps d'évoquer pour nous, ne serait-ce que le temps d'un frisson, l'interdit si fascinant.

La liberté du conte est, en effet, telle que le mariage semble parfois très compromis. On peut ajouter à la vision globale esquissée par MarieLouise von Franz que le héros, pour mieux être à même de continuer le rôle de son père, doit nécessairement passer d'un stade d'enfant au statut d'adulte. Ce parcours très complexe s'achève dans les sociétés primitives par des rites d'initiation au terme desquels le garçon devient définitivement homme et la fille définitivement femme. Or les contes semblent bien avoir gardé quelque chose de ces rites de passage. Le héros-enfant, ignorant encore tout des choses du sexe, ne peut atteindre

\footnotetext{
${ }^{1}$ Le conte de Qamar al-Zaman et Budur est explicite à ce sujet comme nous l'avons exposé dans Le Secret des Mille et Une Nuits ou l'inter-dit de Chéhérazade, Toulouse, 1987.

${ }^{2}$ Franz von, Marie-Louise, L'Interprétation des contes de fées, Paris, La Fontaine de Pierre, 1978, pp. 70-71.
} 
son statut d'adulte qu'en traversant certaines étapes initiatiques qui le conduisent au vrai mariage.

\section{Une initiation nécessaire}

Sur sa longue et difficile route initiatique, le héros connaît des situations très différentes les unes des autres, toutes profondément marquées par le désir, le fantasme, voire l'interdit. Son parcours initiatique est donc non pas tant d'éviter l'interdit ou le désordre, mais plutôt de savoir un jour y renoncer et faire sienne la Loi sans laquelle le groupe sociale ne pourrait perdurer. C'est ainsi que l'ordre social et moral est très souvent rétabli dans des contes qui, l'espace de quelques nuits troubles, ont pu nous égarer dans les sentiers de l'interdit. Il ne s'agit donc pas seulement de voir dans la lecture des contes la description d'une passion amoureuse, légitime ou illégitime d'ailleurs. A dire vrai, le conte ne s'embarrasse pas de morale. Une telle approche n'épuiserait d'ailleurs pas toute la dimension symbolique que peuvent receler ces récits. Aussi sommes-nous convaincus que les contes d'amour disent infiniment autre chose. Car l'amour dont il est question, n'est pas seulement un processus psychologique individuel (comme de nos jours, il peut être mis en valeur), mais possède une fonction sociale de la plus grande importance qui peut être celle de la contestation d'un discours devenu trop normatif. Les Mille et Une Nuits peuvent ainsi servir de mode d'expression dans lequel le désir est affirmé et valorisé au détriment de la loi. L'amour qui y est mentionné pourrait bien être l'expression d'un souhait à défaut d'être la réalité.

Suivre l'éclosion et la résolution de la passion amoureuse des héros est une lecture qui mérite d'être faite en soi, car toute description du dédale amoureux de l'autre reflète quelque chose de ma propre complication, de mon dédale intérieur. Mais ce regard n'est permis qu'à condition de ne pas investir les personnages d'une psychologie qu'ils 
n'ont pas. Car tout le problème est là. Les héros des contes sont-ils vraiment des personnages? Ou ne restent ils pas essentiellement des êtres de papier dont l'épaisseur est celle du rêve ? Car ces figures de personnages posent-elles vraiment la question du sujet ? Ou bien le héros est-il plutôt l'illustration, plus ou moins fidèle et probante d'une idée, d'un rite, d'une initiation dans laquelle il n'y a pas encore réellement de sujet au départ, mais un individu indifférencié qui se met en marche pour atteindre précisément le statut d'homme adulte ? C'est alors que le conte se fait vraiment initiation et que l'amour érotique se confond avec le savoir absolu que procurent les quêtes les plus difficiles. Amour et voyages deviennent alors indissociables. Il arrive encore que l'amour transforme tellement les amoureux qu'ils en sont métamorphosés. Les multiples métaphores qui peuvent peindre l'amour aboutissent ainsi à la métamorphose même du héros. Mais suivons à présent quelques grandes illustrations de l'amour dans les Mille et Une Nuits.

\section{L' amour fatal ou le coup de foudre}

Une des premières caractéristiques de l'amour dans les contes est d'être immédiat. Le conteur ne s'arrête jamais sur la naissance lente et difficile ou conflictuel d'un amour. Celui-ci est direct et son intensité immédiate ${ }^{1}$. C'est particulièrement le cas du jeune prince Qamar alZamân, qui, par le truchement de trois éfrits, se trouve couché à côté de Budur. Dès que ceux-ci le réveillent, il se sent rapidement envahi par ce penchant qu'il a jusqu'alors violemment combattu, allant jusqu'à refuser catégoriquement par trois fois tout mariage proposé par son père.

"A cette douce sensation, le jeune homme ouvrit les yeux, mais les referma aussitôt d'éblouissement et d'émotion. Et il sentit

${ }^{1}$ Cette caractéristique se retrouve dans toute la poésie dite courtoise. L'étude de Tahar Labib Djedidi, La Poésie amoureuse des Arabes, le cas des Udhrites, Alger, Sned, 1970 , pose parfaitement le problème. 
contre lui ce corps plus tendre que le beurre et cette haleine plus agréable que le parfum du musc. Aussitôt sa surprise fut extrême, mais non dénuée d'agrément, et il finit par lever la tête et considérer l'incomparable beauté de cette adolescente qui dormait, inconnue à ses côtés... Donc Kamaralzaman mit ses lèvres sur les lèvres de Sett Boudour et leur prit un long baiser; et, comme elle ne se réveillait pas, il en prit encore un second puis un troisième, sans qu'elle eût marqué le moindre sentiment. Alors il se mit à lui parler : disant : $O$ mon coeur, ô mon oeil ! ô mon foie ! Réveille-toi ! Je suis Kamaralzaman. Mais la jeune fille ne fit pas un seul mouvement. Alors Kamaralzaman, voyant l'inanité de son appel, se dit : Je ne puis plus attendre! il faut que je pénètre en elle, tout $m^{\prime} y$ pousse ! J'essayerai, pour voir si je puis réussir, pendant qu'elle dort"1.

Les éfrits, nous le savons, endorment vite le jeune prince et réveillent à son tour Budur qui, elle-aussi, a toujours refusé ses prétendants. Elle trouve Kamaralzaman à ses côtés. "... dès le premier regard qu'elle lui jeta, elle ne tarda pas à passer de la frayeur à l'admiration et de l'admiration au plaisir et du plaisir à un épanchement de joie qui atteignit bientôt au délire... Et la jeune fille enfouit sa tête sous le bras de l'adolescent, et câlinement le mordilla au cou et à l'oreille, mais sans résultat. Alors ne pouvant plus résister à la flamme allumée en elle pour la première fois, elle se mit de la main à fureter entre les jambes et les cuisses du jeune homme et les trouva si lisses et si pleines qu'elle ne put empêcher sa main de glisser sur leur surface... Après quoi Sett Boudour couvrit de baisers son ami endormi, sans laisser un seul endroit sur lequel elle n'eût imprimé ses lèvres... et, dans cet enlacement, membre contre membre et leurs haleines mêlées, elle s'endormit en souriant" 2 . 
C'est aussi la même chose pour Miryam ${ }^{1}$, qu'un marchand cherchait à vendre au souk, qui tombe amoureuse à la vue du jeune Nour: "Or c'est alors seulement que tu aperçus le jeune Nour, $\hat{o}$ merveilleuse, et qu'à sa vue tu sentis le désir te mordre le foie et l'amour te bouleverser les entrailles : Et tu t'arrêtas soudain, et tu dis à ton maître : c'est celui-là que je veux ! Vends-moi à lui" 2 . Lorsque Hasan voit pour la première fois repartir la djinniyya qui deviendra plus tard sa femme, il resta "stupéfait, les suivait des yeux et, bien longtemps après qu'elles eurent disparu, il continua à fixer l'horizon lointain, en proie à la violence d'une passion que n'avait jamais allumée en son âme la vue de toute autre fille de la terre. Et les larmes de désir et d'amour coulèrent le long de ses joues... Et il continua à soupirer de la sorte, sans fermer l'oeil, jusqu'au lever du soleil. Puis il descendit sur le bord du lac, et se mit à errer de-ci delà, respirant dans l'air frais les effluves qu'elles avaient laissées. Et il continua à se consumer tout le jour dans l'attente de la nuit, pour remonter alors sur la terrasse, espérant le retour des oiseaux. Mais rien ne vint cette nuit-là ni les autres nuits. Et Hassan, désespéré, ne voulut plus ni manger, ni boire, ni dormir, et ne fit que s'enivrer de plus en plus de sa passion pour l'inconnue. Et de cette façon, il dépérit et jaunit ; et ses forces peu à peu l'abandonnèrent, et il se laissa tomber sur le sol, se disant : la mort est encore préférable à cette vie de souffrance" 3 .

Parfois les jeunes amants sont dans l'impossibilité de s'approcher l'un de l'autre bien qu'ils se voient de loin. Leur amour mutuel n'en est

\footnotetext{
${ }^{1}$ Mardrus rapporte ce conte : Histoire du jeune Nour avec la Franque héroüque, vol.2, pp. 259-296.

${ }^{2}$ Mardrus, Histoire du jeune Nour, vol. 2, p. 272. On retrouve une scène similaire dans le conte de la belle Zoumourroud. Quand celle-ci aperçoit Alishar, "l'aspect du jeune homme l'enflamma subitement du plus violent amour... et dit : $O$ crieur, c'est ce jouvenceau-là que je veux, celui au visage gentil, à la taille onduleuse..." vol. 2, p.747.

${ }^{3}$ Mardrus, Les aventures de Hassan al-Bassri, vol. 2, p. 152.
} 
pas moins intense et soudain. C'est ainsi que Ward fi-1-Akmam et Uns al-Wujûd ne pouvant se dire mutuellement leur flamme, s'écrivent des billets d'amour jusqu'à ce que le père du jeune homme découvre le subterfuge et exile alors son fils sur une haute montagne ${ }^{1}$.

Il serait trop long de mentionner tous les cas où les jeunes gens, soit la fille soit le garçon, éprouvent un amour sans transition pour celle ou celui que l'un ou l'autre rencontre et voit physiquement. Mais point n'est nécessaire parfois pour le héros ou la héroïne de voir son partenaire de ses propres yeux. Une représentation, un dessin, une broderie suffit pour que l'amour se déclare aussi vivement que si i:personne était présente. Dans le conte d'Ibrahîm et Jamîla, le jeuni homme découvre une merveilleuse gravure dans un livre qu'il avait acheté à un marchand du souk. Cette gravure n'est rien d'autre que l'image de Jamîla que le jeune Ibrahîm veut à tout prix retrouver. La passion pour cette fille inconnue dont il n'a qu'un reflet, est suivie de tous les effets traditionnels de la passion : pleurs, soupirs, évanouissement fréquents, perte de l'appétit et du sommeil, bref le dépérissement général. Seul le départ et la recherche peuvent lui sauver la vie ${ }^{2}$. C'est également le cas de Sayf al-Mulûk qui tombe amoureux d'une broderie reproduisant l'image de la djinniyya Badi ${ }^{\circ}$ at al-Jamâl. Il part avec son ami d'enfance, $S \hat{a}^{\circ} \mathrm{id}$, après avoir enduré les affres de la passion amoureuse. Son voyage est une suite d'aventures merveilleuses jusqu'à ce qu'il trouve $\mathrm{Badi}^{\circ}$ at al-Jamâl avec laquelle il contacte un mariage monogame.

Le conteur des Mille et Une Nuits franchit une étape de plus lorsqu'il évoque l'éclosion de l'amour, non seulement à partir de la vue réelle ou fictive, mais de l'ouïe. Il suffit à certains héros d'entendre parler de la bien-aimée pour qu'il sentent les effets de l'amour. Qamar al-Zaman, le fils d'un grand marchand du Caire entend un jour un

\footnotetext{
1al-maktaba l-thaqâfiyya, vol. 2, pp. 431-455.

2al-maktaba l-thaqâfiyya, vol. 4, pp. 360-377.
} 
derviche parler d'une certaine Halima qui lui ressemble comme deux gouttes d'eau. Aussitôt il en est amoureux et n'aura de cesse qu'au moment où il la retrouve à Basra ${ }^{1}$. L'amour naissant par simple ouiedire se retrouve dans le conte de Jullanâr et Badr Bâsim ${ }^{2}$. Ce dernier surprend en effet sa mère Jullanâr et son oncle Sâlih s'entretenir sur un éventuel mariage du jeune homme. A peine citent-ils une certaine Jawhara, créature sous-marine, fille du djinn Samandal, que Badr, aussitôt l'aime. "Et Sourire de Lune feignait de dormir, mais se délectait en son âme et se trémoussait en pensée de l'espoir de posséder bientôt cette princesse marine si pesante et si fine !... Quant à Sourire de Lune il se leva sur son séant, comme s'il n'avait rien entendu, et s'étira tranquillement ; mais, en son intérieur, son coeur brûlait d'amouŕ et grésillait comme sur un cendrier rempli de charbons ardents" 3 . Le lendemain, il part en compagnie de son oncle pour rencontrer Jawhara.

Ces quelques exemples de contes nous montrent aisément que l'amour est décrit essentiellement comme une fatalité. Les amants n'y peuvent rien. L'amour les saisit de près ou de loin, l'objet de leur amour étant réel ou simplement représenté. Parfois, une simple parole suffit pour déclencher ce que les moralistes arabes et musulmans ont traditionnellement considéré comme une maladie. Jâhiz (m. 869) le définissait ainsi le ishq passionnel: "Le ishq est un mal contre lequel on ne peut se prémunir; il est semblable aux maladies accidentelles que seul un régime (approprié) permet de repousser... Le ishq se compose de l'amour-sentiment (hubb), de la passion (hawa), de l'affinité ou sympathie (mushâkala) et de la fréquentation (ilf); il débute, s'aggrave, s'arrête à son paroxysme, puis décroît progressivement jusqu'à sa totale

\footnotetext{
1 al-maktabat al-thaqâfiyya, vol. 4, pp. 391-434.

2al-maktaba al-thaqâfiyya, vol. 3, pp. 401-439.

${ }_{3}$ Mardrus, Histoire de Fleur-de-Grenade, vol. 2, p.p. 75-76.
} 
décomposition..."1. Très tôt dans l'islam on s'interroge donc sur cette étrange manifestation qui perturbe les sens de hommes. Un demi siècle plus tard, al-Tawhîdi (932-1012) n'est pas plus positif : "Quant d l'amour (qui vous attache à quelqu'un), il est comme la passion ('ishq), l'affection (mahabba), le kalaf (être épris de quelqu'un), le shaghaf (amour violent), le tatayyum (être esclave de sa passion), l'amour fou (tahayyum), la passion (hawa), l'ardente affection (sababa), le tadanu (être continuellement malade), le tashaji (être affligé). Tout cela n'est que maladie, ou du moins ressemble à des maladies dont sont atteints indifféremment les personnes faibles et les personnes fortes. Il n'y a pas une ombre de raison dans l'amour, ni la marque d'une personnalité..."2. Des hommes comme Ibn al-Jawzi (116-1200), Ibn Qayyim al-Jawziyya (1292-1350) et surtout Ibn Taymiyya (m. 1328) seront encore plus critiques envers l'amour. Et pourquoi le sont-ils, sinon parce que cette forte passion peut conduire tout droit au désordre social.

\section{L'amour adultère}

En effet, dans les Mille et Une Nuits les amants ne manquent pas d'enfreindre les lois sociale. C'est par exemple Zayn al-Mawâsif ${ }^{3}$, jeune musulmane mariée à un Juif. Elle trompe son mari avec son amant Anis qu'elle fait même inviter à la maison, poussant l'hypocrisie jusqu'à faire semblant, devant son mari, de ne pas connaître cet Anis que son mari trouve si charmant : Mais "Anis et Zein al-Mawassif se gardèrent bien, en se voyant, de laisser paraitre qu'ils se reconnaissaient. Et Anis, durant tout le repas, tint les yeux baissés fort honnêtement ; et il feignait une grande discrétion, et ne regardait que le mari. Et le juif en

1Pellat, Charles, "Les esclaves-chanteuses de Gahiz" in Arabica, t. X, 1963, pp. 138138.

2Bergé, Marc, "Une anthologie sur l'amitié d'Abu Hayyân al-Tawhidi", in Bulletin d'Etudes Orientales, Damas, t. XVI, 1959, p. 169.

${ }^{3}$ Mardrus, vol. 2, p. 244. 
lui-même pensait : Quel jeune homme excellent !... Et Anis revint le lendemain, et le jour suivant; et chaque fois, il se comportait, en tout, avec un tact et une discrétion admirable". Zayn al-Mawâsif doit être particulièrement séduisante, puisqu'un patriarche et ses quarante moines en tombent amoureux. "Or le patriarche Danis, embrassé d'amour par la beauté et les charmes de Zein al-Mawassif, ne savait comment s'y prendre pour lui déclarer sa passion. Et la chose était, en effet, bien ardue. Enfin il crut trouver le bon moyen, qui fut d'envoyer à l'adolescente le moine le plus éloquent d'entre les quarante moines du monastère. Et ce moine arriva auprès de l'adolescente, dans l'intention de parler en faveur de son patriarche. Mais, à la vue de cette lune de beauté, il sentit sa langue se lier de mille noeuds dans sa bouche, et, au contraire, son, doigt du ventre parler éloquemment sous sa robe, en se soulevant comme une trompe d'éléphant. Et à cette vue, Zein al-Mawâssif se mit à rire de tout son gosier avec Houboub, Khouboub, Soukoub et Roukoub. Puis voyant que le moine, sans parler, restait avec son outil en l'air, elle fit signe à ses suivantes, qui aussitôt se levèrent et le poussèrent hors de la chambre" 1 .

Dans le conte de Qamar la-Zaman et Halima, cette dernière est mariée à un joaillier de Basra, 'Ubayd. Elle tombe amoureuse de Qamar, dès que son mari lui en parle et s'arrange alors pour que le jeune homme soit invité chez eux et prié de passer la nuit à la maison. Aussitôt la nuit venue, elle passe à l'action après avoir fait boire un narcotique puissant à son mari et au jeune homme. Alors "elle commença par s'asseoir tout contre lui, et se mit à lui caresser doucement le visage avec la main. Et soudain cette poulette affamée se jeta goulûment sur le jouvenceau et se mit à lui becqueter les lèvres et les joues si violemment que le sang en jaillit. Et ces becquées cruelles durèrent un certain temps et furent remplacées par de tels mouvements qu'Allah seul pouvait savoir ce qui pouvait bien se passer sous de telles agitations de la poulette à califourchon sur le jeune coq

${ }^{1}$ Mardrus, Les amours de Zein al-Mawassif, vol. 2, p. 249. 
endormi" 1 . Plus tard, elle s'arrange de telle manière que son mari installe l'amant dans la maison mitoyenne à la sienne. Ainsi Halima, comme dans le Miles Gloriosus de Plaute, fait creuser un tunnel qui lui permet de rejoindre rapidement Qamar sans que ${ }^{\circ}$ Ubayd le sache. Elle se moque ainsi de lui qui ne comprend plus rien, croyant à plusieurs reprises de voir sa femme chez Qamar. "Quant à Halima, passant par l'armoire, elle vint rejoindre Kamar, et lui dit : Tu vois qu'il n'y a pas moyen d'ouvrir les yeux à ce père de la barbe honteuse ! Il ne nous reste donc plus qu'à nous en aller d'ici sans retard" 2 . C'est ce qu'elle fera en emmenant le jeune homme dans sa patrie d'origine Le Caire. Mais de retour auprès des siens, Qamar est désavoué par le père qui lui demande de renoncer à son amante qui est emprisonnée. Aussitôt le jeune homme se sépare de Halîma et épouse la fille du cadi. Après avoir décrit avec force détails les amours interdites entre cette femme mariée et Qamar, le conte devient subitement très moral. En effet, le mariage est à peine terminé que ${ }^{\circ}$ Ubayd arrive au Caire et est reconnu par Qamar qui l'invite à la maison. Le mari trompé retrouve ainsi sa femme infidèle qu'il ne tarde pas de tuer sans que personne n'intervienne. La famille de Qamar donne alors au justicier la soeur même de Qamar en mariage. Ainsi l'ordre social et l'ordre moral sont à nouveau respectés.

\section{L'amour courtois}

Si Halima meurt, punie d'avoir vécue une vie si scandaleuse, d'autres héros meurent par amour et par fidélité. Qamar renie son amante dès l'instant où son père, incarnation de la Loi, le lui demande. En revanche, Ali Ibn Bakkar ${ }^{3}$ réagit tout autrement. Comme ce conte est celui qui se rapproche le plus de l'amour courtois que chantaient les

\footnotetext{
${ }^{1}$ Mardrus, IIistoire de Kamar el de l'experte Ilalima, vol. 2, p. 426.

2 Mardrus, Histoire de Kamar et de l'experte Ilalima, vol. 2, p. 431.

3 al-maktaba al-thaqâfiyya, vol. 2.
} 
poètes udhrites, nous nous arrêterons davantage à cette mise en prose de ce thème.

Le conte d'Ali Ibn Bakkâr est sans doute le plus représentatif des contes dans les Mille et Une Nuits où l'amour courtois est le mieux explicité. Il est aussi le conte où les citations en vers relatifs à l'amour sont les plus nombreuses, à tel point que l'on peut se demander si le conte n'a pas été forgé en fonction des vers qu'il contient ! On compte en effet pas moins de 88 vers pour 37 pages dans l'édition de Dâr al-tawfîq. Le conte de Masrûr et Zayn al-Mawâsif qui a 61 pages contient trente citations en vers, soit 223 vers ! Le nombre impressionnant de ces vers d'amour cités dans Ali Ibn Bakkâr et Shams al-Nahâr mérite réflexion. Pourquoi le conteur utilise-t-il autant la poésie?

Une première remarque peut être faite à propos de la citation ellemême. Nous savons que très souvent, la citation du Coran et du hadîth authentifie la littérature classique et lui donne des lettres de noblesse incontestables. Elle la fait participer au sérieux dont le Coran et le hadîth sont naturellement chargés. De même, la citation des vers, dans une tradition orale, moins noble, joue un rôle semblable. Les vers qui appartiennent à la littérature "sérieuse", authentifient en quelque sorte cette littérature orale populaire, et lui donnent un éclat supplémentaire, en la rapprochant de la littérature officielle. En somme, les vers, dans les contes jouent un rôle semblable à celui des versets du Coran dans la littérature de l'adab. Car le problème est toujours bien celui-là : comment légitimer un texte ? Si Le Coran se justifie par lui-même, et il est le seul, tous les autres textes se justifient en ce sens qu'ils explicitent le sens ou le contenu du Coran. La science classique comme la grammaire, la philologie, l'histoire, la géographie... et l'adab même ne sont rien qu'une extension du Coran. On peut dire par analogie que les contes érotiques et principalement celui d'Ali Ibn Bakkâr sont une mise en prose de vers chantant l'amoureux courtois.

Les vers du conte d'Ali Ibn Bakkâr ont également un pouvoir évocateur tel que les amants s'évanouissent en les entendant. Ali Ibn Bakkâr mourra même en entendant la récitation de vers d'amour. Jamais 
ailleurs les citations poétiques n'ont un effet aussi fort. On comprend mieux cette mise en scène qui par sa répétition peut paraître artificielle, surtout à un lecteur occidental qui connaît une autre sensibilité, si on la replace dans le cadre du tarab. Le tarab est en effet une intense émotion que déclenche l'effet de la beauté des vers ou des chants. Certains mystiques y recouraient pour provoquer l'expérience mystique, tel Jalâl al-Dîn al-Rûmi. Ils faisaient appel à la musique, au chant, à la poésie voire même à la contemplation d'un beau jeune homme pour entrer en transe et ainsi accéder à l'union mystique avec le divin. L'amoureux de l'amour courtois se trouve dans une situation semblable. Il ne rêve que d'union et de présence de l'être aimé. Les vers d'amour ne font que mettre un peu plus à nu les sentiments qui le brûlent. Cette sensation peut aller chez certains jusqu'à l'évanouissement.

Le conte d'Ali Ibn Bakkâr est le seul également qui se termine vraiment dramatiquement et tragiquement par la mort des amants. Mais il ne s'écarte pas totalement de certaines règles conventionnelles propres à l'amour courtois tel qu'il est dépeint dans les Nuits. Ce conte du cycle bagdadien est construit sur une intrigue simple, ordonnée selon un plan chronologique que l'on peut diviser en trois étapes, sans qu'il y ait la typique construction en abîme propre aux Nuits. Elles marquent chaque fois une rencontre entre les deux amants, et renforcent chaque fois un peu plus l'impossible amour ; la dernière étape illustre la séparation finale.

Remarquons d'emblée que ces trois étapes sont marquées par des espaces clos. Les deux premières sont en même temps exposées à une circulation intense. La boutique du marchand devient ainsi très vite impropre à des rendez-vous répétés en raison du passage des clients éventuels. Le palais du Calife est plus dangereux encore, puisque la présence du chef suprême est à craindre à tout instant. Seul le troisième espace, un espace neutre, pourrait garantir les amours des amants. Mais l'amour courtois complique la situation. 
La première rencontre entre Ali Ibn Bakkâr et Shams al-Nahâr se fait dans la boutique de l'ami du jeune prince Abu l-Hasan dont le père, riche marchand, était un habitué du palais califal. Ce détail (absent chez Mardrus) explique pourquoi Shams al-Nahâr se rendait à la boutique. En effet c'est là que le fils, Abu l-Hasan, vendait aux favorites du Calife les étoffes et les pierreries qu'elles désiraient.

Le coup de foudre est une caractéristique de ce genre de contes. Et Ali Ibn Bakkâr n'y fait pas exception. En effet, dès qu'il aperçoit la favorite du Calife, il est troublé par la jeune visiteuse (salabat $\left.{ }^{\circ} a q l a h u\right)^{1}$. Et Shams al-Nahâr semble bien l'avoir remarqué aussi puisqu'elle avertit Abu 1-Hasan qu'elle lui enverra une servante pour ramener le jeune prince auprès d'elle. C'est ce qui arrive en effet peu de temps après (ba ada sầ atin jấat al-jâriyya) (p. 73).

A peine Shams al-Nahâr fut donc revenue au palais califal, car elle était la favorite de Harûn al-Rashîd, qu'elle envoya par sa servante un billet au prince Ali pour l'inviter chez elle. Dès cet instant, Abu 1-Hasan, le riche marchand de Bagdad que fréquentait Ali Ibn Bakkâr, prince d'origine perse, se fera l'intermédiaire entre ce dernier et la favorite du Calife.

Quand Shams al-Nahâr et Ali Ibn Bakkâr sont de nouveau en présence l'un de l'autre, dans les appartements de la favorite du Calife, ils se mettent à déclamer des vers. Ali Ibn Bakkâr fond en larmes, rempli d'émotion, et Shams al-Nahâr, à ce spectacle, est envahie par la passion amoureuse (ahraqaha al-wajd wa-l-ghurâm wa ttalafaha l-walah wa-lhayâm) (p. 75). En s'étreignant, ils tombent tous les deux évanouis ! Abu l-Hasan est effrayé de la passion de son ami (ya sayyidi ma balgha bika l-hawâ) (p. 75). Quant aux deux amants, ils se mettent alors à verser d'abondantes larmes (p. 76). Puis ils passent la journée ensemble dans la joie de vivre, quand soudain Harûn al-Rashîd annonce sa visite.

Cette seconde rencontre se fait donc sur l'initiative de Shams alNahâr au palais même du Calife, qui, au bout d'un certain temps, fait

1al-maktyaba al-thaqâ̂fiyya, vol. 2, p. 73 . 
annoncer sa venue impromptue. Le prince Ali et son ami Abu 1-Hasan sont alors dissimulés derrière une fenêtre d'où ils peuvent voir la rencontre du Calife et de la favorite. Cette scène met en relief la douleur du jeune prince qui se transforme en quelque sorte en voyeur impuissant de l'amour d'un rival. Une chanteuse, connaissant l'amour de sa maîtresse pour le jeune prince, se met à déclamer des vers d'amour dans lesquels Shams al-Nahâr naturellement se reconnaît. L'effet de la poésie, en arabe le tarab, est tel que la jeune favorite en est bouleversée : "Lorsque la belle Schamsennahar eut entendu ces vers, elle fut pénétrée d'une émotion si vive qu'elle se renversa sur son siège et tomba évanouie entre les bras de ses femmes qui avaient volé à son secours. A cette vue, le prince Ali, qui, dissimulé derrière la fenêtre, regardait cette scène, avec son ami Ben-Taher, fut tellement saisi de douleur sympathique qu'il tomba également tout de son long évanoui dans les bras de son ami Abalhassan ben-Taher"1.

L'effet du tarab, provoqué par la musique ou la poésie ou encore les deux combinés, n'est donc pas une émotion ordinaire. Il est généralement accompagné de pleurs. Ceux-ci coulent alors à flot si la bien aimée est absente. Le conte ici pousse au paroxysme l'effet des vers d'amour. Dans les autres contes le tarab est moins fort. Il provoque douleur et plaisir mais laisse les amants conscients d'eux-mêmes. C'est dans cet état de passion intense qu'Abu-l-Hasan ramène à la maison son ami Ali Ibn Bakkâr.

De retour du palais, Abu l-Hasan recueille l'amoureux chez lui tellement il est affecté par l'amour de Shams al-Nahâr (p. 81). Il doit constater aussi que l'amour continue son effet ravageur. Pour le consoler de l'absence de sa bien-aimée, Abu l-Hasan lui récite des vers d'amour, mais lorsqu'il les entend, son mal ne fait qu'augmenter : "A ce chant, le prince Ali, déjà si faible, fut dans un tel état d'anéantissement, causé par les souvenirs intenses qui lui revenaient à la mémoire, qu'il se mit à pleurer de nouveau". Le texte arabe stipule qu'il s'évanouit

${ }^{1}$ Mardrus, vol. 2, pp. 524-525. 
jusqu'à l'aube (p. 82). De son côté, Shams al-Nahâr s'évanouit en présence du Calife et demeura ainsi la moitié de la nuit (p. 82).

Abu 1-Hasan, qui entre temps était revenu à la boutique, rencontre une servante du palais qui veut être conduite auprès de Shams al-Nahâr. Ce que fait Abu l-Hasan (p. 86). Grâce à cette servante les deux amants peuvent échanger des lettres d'amour qui augmentent leur passion (pp.86-87). Cette intensité fait peur à Abu 1-Hasan qui décide de fuir à Basra non sans avoir recommandé Ali Ibn Bakkâr à un de ses amis qui désormais en prendra soin (pp. 88-90). Le conte introduit dès lors un nouveau confident capable de maintenir le lien entre Ali Ibn Bakkâr et Shams al-Nahâr (p. 93). Le nouvel ami ne tarde pas à louer une maison à côté de la sienne pour arranger plus facilement les rencontres entre les deux amants (p. 95).

La troisième et dernière rencontre s'effectue en effet dans une maison que le confident fait spécialement installer pour recevoir les amants. C'est un espace, en apparence neutre, qui ne comporte pas les dangers de la boutique, ni ceux du palais. De plus, il jouxte celui du protecteur. Les conditions sont donc réunies pour que les rendez-vous restent clandestins et ignorés de tous. Les amants sont donc en sécurité, personne ne peut les dénoncer. Mais nous devinons d'avance que ce schéma sied pour des amours ordinaires. L'amour courtois ne se justifie que dans la difficulté voire l'impossibilité. Il faut donc aussi que cet espace-là soit piégé d'avance. L'intérêt est seulement de savoir comment et par qui.

La première nuit que les amants passent dans cette maison, ils sont assaillis par des voleurs qui les emmènent avec eux (p. 98). Et, au petit matin, un complice attend l'ami d'Abu l-Hasan qu'il entraîne jusqu'auprès des voleurs car ceux-ci voulaient l'interroger sur la qualité des deux prisonniers : "... (il) vit assis, immobiles, dix hommes de même habillement et de figures tellement ressemblantes et si absolument identiques qu'ils s'imagina voir une seule énorme figure 
répétée dix fois par des miroirs"l. Amîn révèle alors l'origine princière des amants, ce qui décide les voleurs à leur rendre leur entière liberté ${ }^{2}$.

Cette épreuve n'est somme toute qu'un incident banal pour les amoureux, en comparaison de ce qui les attend plus tard. Mais remarquons déjà que les amants sont incapables de vivre leur amour comme ils le veulent, ni dans la boutique, ni dans le palais, ni même dans un endroit neutre : la maison. Chez le marchand, ce sont les clients qui perturbent les plans des amoureux, au palais ce sont les multiples servantes et serviteurs et surtout le Calife lui-même, dans la maison ce sont finalement les brigands qui compromettent le projet courtois. Ces trois espaces démontrent donc en quelque sorte l'incapacité totale de donner libre cours au sentiment et à la passion, comme si aucun endroit au monde ne pouvait désormais recevoir impunément les amants. L'amour ne peut être vécu ni chez l'amant, ni chez l'amante, ni même chez quelqu'un d'autre. Cette impasse ne peut donc que se solder par une tragique séparation.

\section{Les effets de l'amour}

Ali Ibn Bakkâr tombe amoureux dès le premier regard porté sur Shams al-Nahâr : "Or à peine le jeune prince Ali ben-Bekar eut-il aperçu ce visage qu'il en fut frappé d'admiration, et une passion s'alluma au fond de son foie..." 3 . Le caractère immédiat de l'amour, véritable coup de foudre est partagé par Shams al-Nahâr dès qu'elle entend Ali Ibn Bakkâr réciter des vers : "Lorsque l'adolescente eut entendu ces vers récités avec un accent désespéré, elle fut charmé du

\footnotetext{
1 Mardrus, vol. 2, p. 538.

${ }^{2}$ Les dix voleurs peuvent rappeler les dix voleurs du célèbre roman gre-alexaandrin de Daphnis et Chloé.

${ }^{3}$ Mardrus, vol. 2, p. 517.
} 
sentiment délicat qui les inspirait, et elle fut plus vivement subjuguée par l'air charmant de son amoureux" (idem).

L'amour courtois est généralement suivi d'effets incontrôlables. Il envahit les êtres malgré eux, s'en empare et en fait de lentes mais inexorables victimes. Celui qui en est atteint rentre ainsi dans un état second et cesse d'appartenir au monde des simples mortels. Ainsi, "quant à Ali ben-Bekar, une fois l'adolescente partie, il resta un bon moment à ne savoir plus ce qu'il disait, et tellement qu'Abalhassan fut obligé de l'avertir que les clients remarquaient son agitation et commençaient à s'en étonner. Et Ali ben-Bekar répondit : $O$ benTaher, comment ne serais-je pas agité et, moi-même, étonné de voir mon âme chercher à s'échapper de mon corps pour aller rejoindre cette lune qui oblige mon coeur à se donner sans consulter mon esprit ?" (idem). Nous devinons aisément dans cette première approche les symptômes de la folie qu'un Majnûn a si hautement incarnée. L'esprit est perturbé par l'intensité de l'amour. Et l'on peut même dire à la suite de l'étude d'André Miquel $^{1}$ à propos précisément de la merveilleuse figure de Majnûn que l'amour courtois dans sa forme exacerbé doit aboutir à la folie et à la mort.

Mais pourquoi les amoureux courtois ne combattent-ils pas leur amour qui les fait tant souffrir ? Pourquoi ne font-ils rien de positif pour y répondre vraiment?

L'amour s'empare des amants et en fait des victimes passives. Et ce qu'ils entreprennent augmente généralement cette impossibilité d'un amour véritable. Ainsi quand Shamas al-Nahâr invite Ali Ibn Bakkâr au palais, ce n'est pas l'endroit idéal pour une expression amoureuse. Lf danger, d'ailleurs, ne tarde pas à se manifester puisque très vite Harûr al-Rashîd annonce sa visite. Il faut encore que ce soit l'ami d'Abu 1 Hasan qui ait l'idée de louer une maison, alors que cette question auraj pu être réglée par le jeune prince lui-même. Tout se passe comme si l

${ }^{1}$ Miquel, André et Kemp Percy, Majnûn et Layla, l'amour fou, Paris, Sinbad, 1984 Miquel, Majnûn, l'amour poème, Paris, Sinbad, 1984. 
passion paralysait les amants et les rendait incapables d'arriver à leur but. Mais en fait, n'attendons pas des amoureux courtois qu'ils deviennent actifs, leur action irait à l'encontre de ce qu'ils veulent vraiment : souffrir pour avoir le sentiment d'aimer, et pour cela il faut renoncer à tout ce qui mettrait véritablement en danger leur sentiment et leur état amoureux.

C'est cette tension négative qui caractérise les amants et que ReyFlaud définit non pas comme l'amour courtois mais comme "la névrose courtoise". Car l'amant en somme doit entretenir la douleur pour faire l'expérience de son amour. Fuir la douleur, renoncer à la souffrance, éviter la mort reviendrait à pratiquer un amour quotidien, ordinaire. Les amants courtois sont promis au paradoxe. L'amour qui généralement est source de bonheur, de joie et de satisfaction engendre ici peine, douleur et souffrance. Les amants courtois ne combattent donc pas leur amour mais souffrent et meurent pour lui. Lui l'amour.

L'autre est moins important que l'amour. Car dans l'amour courtois, c'est en effet le sentiment qui prévaut sur la personne. L'amant aime avant tout l'amour et l'autre n'est que le lieu où s'ancre son désir impossible. Le monde cesse d'être l'espace habituel ou se meut l'amant, il ne peut "vivre" que dans l'espace que crée la présence de l'autre. Mais, celui-ci vient-il à disparaître, le monde auquel il a identifié l'espace imaginaire de l'autre, alors s'écroule. Si l'autre était important, l'amant lui laisserait sans doute une autonomie et une indépendance. Or il n'en est rien, la présence de l'autre devient indispensable non pas pour un échange réel avec lui, ou pour d'éventuels projets communs, mais pour entretenir d'une manière plus brûlante l'état présent, comme s'il fallait figer dans l'intensité d'un seul instant ce qui ne peut être vécu que dans le temps et donc dans la déperdition.

Le refus de communion charnelle, le refus d'un engagement concret, la distance entretenue d'un corps à l'autre ne sont que l'expression du refus de se confronter à l'autre, le refus de prendre en compte le réel qui contredit immanquablemont les illusions et les rêves. L'amant courtois a donc essentiellement besoin de se nourrir d'images 
absentes et quand la présence de l'autre se fait trop intense il s'évanouit comme pour nier encore le plaisir charnel qui est en train de le prendre au corps. L'amour courtois ainsi défini est une dramatique volonté d'être à deux pour demeurer en fait seul. La tension qui résulte de cette intention donne à cette démarche inconsciente toute l'intensité dramatique à laquelle sont parfois tentés les amoureux.

On ne compte plus le nombre de fois où les amants pleurent et tombent évanouis. C'est là une conséquence directe de leur amour impossible. Cet évanouissement a valeur de symbole, car il évoque une première forme de mort. On ne s'étonnera donc pas que les amants courtois meurent vraiment. Ils auront fait l'apprentissage de la disparition totale dans les multiples petites disparitions momentanées que sont les évanouissements auxquels ils s'exposent si facilement. En somme l'amoureux, à travers ces évanouissements, apprend déjà d'une certaine manière à mourir définitivement.

Une seconde remarque s'impose dans cette réflexion sur l'amour courtois du conte d'Ali Ibn Bakkâr'1. Pourquoi Shams al-Nahâr est-elle la favorite du Calife, l'autorité suprême du monde arabo-musulman ? Pourquoi le conte se permet-il de contester ainsi l'autorité absolue sur terre ? Certes, déjà Shahrayar a connu la cruelle épreuve d'une femme infidèle. Et d'autres rois dans d'autres contes ont connu la même infortune. Harûn al-Rashîd, le maître du monde, en somme n'est pas le maître des coeurs. Il peut imposer sa puissance aux volontés humaines et les soumettre mais le coeur et les sentiments de ses favorites lui échapperont toujours. Par là, le conte montre la force souterraine de l'amour et du désir. Aucun coeur humain n'est à l'abri du coup de foudre. Et aucun homme ne peut se dire à l'abri d'un bouleversement profond. Harûn al-Rashîd ne peut empêcher sa favorite d'être amoureuse d'un autre. Alors qu'il aurait sans doute tous les moyens pour combler les désirs de Shams al-Nahâr. Lorsqu'il s'agit des choses du coeur, les

${ }^{1}$ Ce conte figure dans l'édition de Beyrouth, al-maktaba al-thaqâfiyya, 1979, pp. 231236. 
biens matériels ne sauraient combler le vide que creuse l'amour. L'amour-passion des amoureux courtois ne se satisfait de rien de matériel. Il leur faut la présence de l'autre, mais cette présence est impossible et toute leur passion vient de cet impossible là. On peut voir aussi dans cette passion amoureuse l'image de la contestation radicale du pouvoir politique. Le Calife est le maître du monde et gouveme tous les sujets sans exception, mais son pouvoir est contesté non de l'extérieur, visiblement, officiellement mais de l'intérieur, d'une manière invisible, sournoisement. L'amour qui court dans le coeur de sa favorite, toujours, lui échappera. Cet aspect contestataire de l'amour udhrite a été développé par Labib Djedidi'.

L'amoureux courtois prend donc une revanche singulière sur le prince ou le puissant en lui ravissant son objet d'amour de prédilection. Il devient ainsi un rival absolu en détournant vers lui, l'amour que le prince croyait régir. L'amoureux courtois, cependant, ne cherche pas à posséder la femme de l'autre. Il ne revendique pas non plus le sexe de celle qui appartient économiquement et politiquement au prince. L'amoureux courtois n'a pas de satisfaction à tromper un mari, fût-il sultan. Sa contestation est peut-être encore beaucoup plus radicale. En refusant le sexe de la dame qu'il aime, il conteste aussi le rôle que joue le prince auprès d'elle. Il rejette ainsi toute identification au prince qui exerce son pouvoir sur l'autre. Refuser le sexe devient ainsi une métaphore grandiose de la contestation de tout pouvoir. L'amoureux courtois n'a de satisfaction, si s'en est une, que dans la fuite et la fragilité du pouvoir. Il lui suffit de savoir que celle qu'il aime appartient à quelqu'un d'autre pour que cet amour vaille la peine d'être vécu dans son impossibilité. Il lui suffit de voir l'autre, l'amante, arrachée au pouvoir du maitre. Et pour que cet arrachement soit total, il se garde bien de se l'attacher par un quelconque commerce sexuel. La chasteté n'est donc pas une vertu morale, car si l'amoureux courtois était vraiment vertueux, entretiendrait-il un amour illicite ? La chasteté est la stratégie

${ }^{1}$ Djedidi, La Poésie amoureuse des Arabes, le cas des Udhrites, Alger, Sned, 1979. 
absolue pour signifier que l'amour doit rester infiniment libre. Une liberté qui ne peut se solder en point final que par la folie ou la mort.

En effet, bientôt Harûn al-Rashîd est mis au courant de l'amour de sa favorite pour Ali Ibn Bakkâr (p. 104). Aussitôt le confident et le jeune prince prennent la fuite hors de Bagdad. Dans leur fuite ils sont victimes de brigands qui les dépuillent de leurs biens (p. 105) non loin de la mosquée d'une oasis. Ali Ibn Bakkâr était épuisé non seulement par la fatigue de cette fuite mais surtout à la pensée de sa bien-aimée : "C'est ici que je vais enfin mourir, puisque Schamsennahar ne doit plus être en vie à l'heure qu'il est !" 1 . Un vieillard qui faisait la prière dans la mosquée accueille les deux hommes chez lui, quand une voix plaintive dans l'oasis chante des vers d'amour, sur la séparation des amants. "Or, à peine Ali ben-Bekar avait-il commencé à entendre ce chant qu'il releva la tête et se mit à écouter, hors de lui. Et quand la voix se fut éteinte, nous le vîmes soudain retomber en poussant un grand soupir: il avait expirê..." 2 .

Son ami revient aussitôt à Bagdad où il rencontre la confidente de Shams al-Nahâr. Celle-ci lui rapporte que Harûn al-Rashîd lui avait pardonné, par amour pour elle. Néanmoins, elle expire en entendant chanter des vers d'amour (p. 108). Dans la version de Mardrus, le confident se met d'accord avec la confidente pour faire enterrer Ali Ibn Bakkâr à côté de Shams al-Nahâr ${ }^{3}$.

Cette fin dramatique d'une part d'un amant qui se laisse mourir à l'idée que l'élue de son coeur est déjà morte, et d'autre part de l'amante qui s'éteint de langueur en pensant à son amoureux, alors qu'elle occupe la place la plus haute de l'échelle sociale, est rapportée avec un dépouillement qui évite tout pathos. Même si les pleurs coulent avec abondance, ils ne submergent jamais l'intensité dramatique de cet amour fatal où la chasteté entre les amants est conservée intacte. Cette fatalité est

$1_{\text {Mardrus, vol. 2, p. } 544 .}$

2 Mardrus, vol. 2, p. 545.

3 Mardrus, vol. 2, p. 547. 
reconnue et acceptée. Elle ne peut avoir de solution heureuse pour les amants. Il ne leur reste qu'à accepter hérö̈quement la mort. C'est dans cette logique qui ne manque pas de grandeur et de vertu que l'Islam a reconnu le statut de martyr à ceux qui mouraient d'amour. Que le hadîth soit vrai ou faux, il témoigne d'une réflexion qui a pris au sérieux cet état d'âme. La souffrance qu'engendrait certaines passions méritait bien que l'amant jouisse du Paradis en récompense de ce qu'il a accepté et enduré sur terre.

Le conte d'Ali Ibn Bakkâr représente donc dans l'ensemble des contes une forme originale de l'amour courtois dont l'idéal a été chanté par toute une veine poétique, et à laquelle l'Arabe est fortement sensible quelles que soient les époques. De cette vision courtoise de la femme et de l'amour, il reste des bribes plus ou moins importantes dans d'autres contes dès l'instant qu'il s'agit de peindre l'amour. C'est ainsi que les Mille et Une Nuits présentent aussi des contes où l'amour entre les hommes et les femmes s'inscrit dans une reconnaissance mutuelle très généreuse et une fidélité absolue.

\section{L'amour fidèle}

Le conte de Hasan al-Basri est un des égyptiens d'origine persane, qui met nettement l'accent sur la fídélité dans l'amour. On peut penser que primitivement ce fut un conte de "fées," tellement il est question de djinns et de merveilleux. L'élément magique l'emporte en effet, très nettement sur les autres thèmes. Le voyage cependant occupe une seconde place, mais il faut ajouter que ce voyage se complique au fur et à mesure que l'on approche de la fin. La première partie : le mariage de Hasan avec la djinniyya est décrit dans une intrigue relativement claire et bien ordonnée, mais la seconde partie : la recherche de sa femme qui s'est échappée lors de son absence, est compliquée et surabonde en données merveilleuses. 
La fidélité de l'homme envers la femme et celle de la femme envers l'homme recoivent ici une note particulière. On apprend de la bouche de la vieille Shahwâ qui parle de Hasan à Nûr al-Hudâ, la soeur de sa femme, que de sa vie, elle n'avait jamais vu un humain aussi amoureux que lui (lam ara 'umri âdamiyan aqwâ qalban minhu... li'anna l-hawâ qad tamakanna minhu ghâyata tamakkun) ${ }^{1}$. Plus tard, quand il aura rejoint sa femme, ils se pardonnent mutuellement leur séparation (p. 76). Manâr al-Hudâ lui avoue qu'une femme ne connaît la valeur de l'homme que lorsqu'elle l'a quitté et Hasan lui demande pardon d'être parti et de l'avoir laissée seule avec sa mère (p. 76). Cette confiance réciproque donne une tonalité très particulière au conte où certaines réminiscences courtoises se font encore jour. Ainsi Hasan perd l'appétit et le sommeil (p. 9), tombe malade et pleure souvent (p. 10). Il s'évanouit de nombreuses fois en se rappelant sa bien-aimée (pp. 25-26). Il manifeste en plus de cela une constance dans la recherche de sa femme qui défie la mort à tout instant. Il ne recule devant aucune difficulté sans hésitation aucune. Malgré les nombreuses péripéties relatées dans le conte, affrontement des éléments de la natures, affrontement des armées de la soeur de sa femme Nûr al-Hudâ,... rien ne le fait reculer, devant le désir absolu de retrouver sa femme et de la ramener chez lui à Bagdad. Il exprime sa fidélité dans l'attachement exceptionnel à sa femme. La première fois qu'il la rencontre, il en est fou amoureux. Mais sa femme ne partage pas tout à fait cette passion. Il faudra les épreuves imposées par sa soeur Nûr al-Hudâ pour que Manâr al-Sanâ s'attache définitivement à son mari, jusqu'à la mort.

Rien ne nous interdit de penser qu'il y a là une critique indirecte à l'institution de la polygamie. Mais une telle critique devient vite une contestation d'un ordre social. Or le conte peut-il se faire impunément le lieu de cette contestation? Il semble que le conteur ait prévu ce blâme en mettant en garde le conteur éventuel. Ces contes ne peuvent être racontés à n'importe qui ! Mardrus place ainsi cette mise en garde, propre au

$1_{\text {al-maktaba al-thaqâfiyya, vol, 4, p. } 53 .}$ 
conte de Sayf al-Mulûk, en introduction à celui de Hasan al-Basril. Quel que soit ce déplacement de l'introduction du conte, il est clair que le conteur avertit son public. Peut-être qu'il y a en effet plus qu'une simple captatio benevolentiae. Il faut avertir le public que le conte est quelque peu subversif et pour que la subversion de l'ordre normal du mariage ne soit pas trop forte, le conteur met dans la bouche des djinns ces propos si dangereux. Tout se passe donc comme si les deux contes étaient suffisamment proches ou suffisamment "subversifs" pour mériter la même introduction restrictive.

Quand Hasan aperçoit la première fois celle qui deviendra sa femme, il comprend le sens de l'interdiction qui lui avait été faite de monter sur la terrasse : "...la plus belle remonta sur l'estrade et alla s'asseoir, n'ayant pour tout vêtent que sa chevelure. Et Hassân en contemplant ses charmes, sentit sa raison s'envoler, et il pensa : Ah! je sais bien maintenant pourquoi ma soeur Bouton-de-Rose m'a défendu d'ouvrir cette porte ! Voici que mon repos est à jamais perdu"2. Quand les filles eurent repris leur manteaux de plumes et furent parties, "Hassân, stupéfait, les suivait des yeux et, bien longtemps après qu'elles eurent disparu, il continua à fixer l'horizon lointain , en proie à la violence d'une passion que n'avait jamais allumé en son âme la vue de toute autre fille de la terre" (idem). Cette passion ne réduit cependant pas Hasan à néant. Contrairement à Ali Ibn Bakkâr, il se montre actif, entreprenant, s'expose aux dangers pour retrouver sa femme. Il est donc loin de se laisser mourir comme le partenaire de Shams al-Nahâr. Héros positif; Hasan incarne l'amant résolu qui est prêt à payer cher le prix de son amour, comme cet autre héros : Sayf alMulûk.

Tout comme le conte de Haşan al-Basri, celui de Sayf al-Mulâk est partiellement persan et l'élément "fantastique" l'emporte sur le voyage

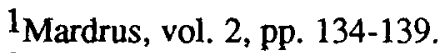

${ }^{2}$ Mardrus, vol. 2, p. 152. 
proprement dit, bien que le conte ne l'ignore pas. Sayf al-Mulûk part à la recherche d'une djinniyya : $\mathrm{Badr̂}$ at al-Jamâl dont il a découvert le portrait cousu dans une veste. Ce conte semble avoir été un récit indépendant au Xe siècle en Perse mais intégré ultérieurement des les Nuits . Sayf alMulûk ne se contente pas non plus d'attendre que vienne à lui celle qu'il aime. Il part avec son ami d'enfance $S \hat{a}^{\circ}$ id et une troupe de vingt mille hommes, comme s'il fallait conquérir l'amour. Mais cette immense armée est peu à peu détruite jusqu'à ce que Sayf al-Mulûk se retrouve seul face à celle qu'il aime. Symboliquement, ce dépouillement de la force coercitive montre que l'amour, peut-être, ne se prend pas mais se donne. Sayf al-Mulûk est dépouillé de toute sa force militaire et devient non pas le sujet de l'amour pour $\mathrm{Badî}{ }^{\circ}$ at al-Jamâl mais en quelque sorte l'objet de cet amour. Il est irrésistiblement ensorcelé par cette fille des djinns à laquelle il demande le mariage hors du commun. Car il est l'illustration d'une union, en apparence impossible, rêvée selon les cas, d'un humain avec une djinniyya. L'union de deux mondes qui, normalement, ne sont pas appelés à communiquer. Cette union entre une djinniyya et un humain peut surprendre un contemporain, mais la tradition arabe ancienne acceptait ces unions comme nous le verrons plus loin. Ibn Hazm traite d'associateur celui qui nie l'existence des djinns ${ }^{1}$.

Le sévère Ibn Taymiyya rappelle qu'il ne saurait y avoir de divergence parmi les Musulmans sur leur existence. Fahd écrit au sujet des relations possibles entre djinns et humains: "Copulation et fécondation étaient considérées possibles entre des djinns et des humains. De ce genre d'unions sont issus Balqîs, reine de Saba et DhulQarnayn dont la mère fut une femme du nom de Fîrâ et le père un ange du nom de ibra..."2. Mais il s'agit, ici, essentiellement de la fidélité exprimée à celle qui est aimée. C'est en tous les cas le thème majeur du conte de Sayf al-Mulûk. En effet, Badîat al-Jamâl, une djinniyya, refuse

1On lira avec intérêt l'étude de T. Fahd, "Génies, anges et démons", Paris, Seuil,1971.

2Fahd, Tawfiq, "Génies, anges et démons", p. 193. 
l'amour de Sayf al-Mulûk précisément parce que les humains sont connus pour leur inconstance. L'amoureux a beau répéter à la djinniyya que Dieu n'a pas créé les humains tous pareils, elle se laisse convaincre avec beaucoup de difficulté. Elle finit par accepter l'amour de Sayf alMulûk avec la promesse de n'être jamais délaissée au profit d'une autre.

$\mathrm{Au}$ fur et à mesure que le conte progresse, nous pouvons remarquer que le récit met l'accent sur la promesse de cette fidélité que Sayf doit faire deux fois. Une autre remarque s'impose : Sayf ne peut arriver jusqu'à $\mathrm{Badî}^{\circ}$ at al-Jamâl par la force de son armée, qui est décimée, ni par sa seule force personnelle. Il doit se faire aider par une fermme. Son succès dépend d'abord d'elle. La réussite de l'amour passe donc essentiellement par la femme. Enfin l'amour est un don réciproque dans lequel personne ne peut s'imposer à l'autre et cet amour exige le respect total et la fidélité sans condition.

Un dernier exemple d'amour et de fidélité peut être perçu dans $l e$ conte de Ghânim Ibn Ayyûub $b^{1}$. Ce conte souligne par ailleurs la constance d'un homme innocent

Ce qui ressort de cette histoire est essentiellement la fidélité à la femme et le respect du statut du mariage. La favorite du calife s'appelle Quwat al-Qulûb, la force des coeurs, et est détestée par sa femme légitime Sitt Zubayda ${ }^{2}$ qui profite d'une de ses absences pour faire disparaître sa rivale. Elle la fait endormir et charge trois Noirs de l'enterrer, enfermée dans une caisse. Mais les Noirs négligeants laissent traîner la caisse et passent leur temps à se raconter l'histoire de leur eunuquat. Un jeune marchand de Basra, Ghânim Ibn Ayyûb aperçoit la caisse et en l'ouvrant découvre la favorite du Calife: Il l'emmène aussitôt chez lui et devient amoureux fou de cette fille admirable qu'il ne connaît

1al-maktaba al-thaqâfiyya, vol. 1, pp. 231-256.

2 Mardrus, vol. 1, p. 278. 
pas encore réellement. Nous retrouvons ainsi le thème de l'amour immédiat et de la passion grandissante de l'amoureux courtois.

Dans un premier temps, Ghânim pense être en présence d'une femme libre, aussi fait-il quelques avances, poussé par le feu de la passion. Ghânim soupire en effet après son amour mais la jeune fille accepte à peine les baisers et les caresses sans jamais aller plus loin : "A cette vue, le désir se mouvementa chez Ghânem, qui dit: $O$ ma maîtresse, me permets-tu maintenant de goûter ta bouche? L'adolescente répondit : Par Allah ! c'est une chose que je ne puis vraiment te permettre, ô Ghânem que j'aime, car il y une chose fort contrariante qui est écrite là sur le cordon de mon caleçon ! Et je ne puis maintenant te le montrer"1. La volonté de demeurer chaste peut être entendu comme un écho lointain de la courtoisie, mais en fait cette chasteté a un sens nouveau. On apprendra le secret du refus de la jeune fille plus loin quand Ghânim se fera encore plus pressant : "O Ghânem, écoute-moi : Je vais enfin t'expliquer ma situation et te faire connaître mon secret... $O$ mon maître Ghânem, lis ce qu'il y a d'écrit sur le bout de ce cordon ! Et Ghânem prit le bout du cordon et vit qu'il y avait brodé dans la trame même en lettre d'or, ces mots d'écriture : Je suis à toi et tu es à moi, ô descendant de l'oncle du Prophète :" 2 .

C'est ainsi que Ghânim apprend que Quwat al-Qulûb était la favorite de Harûn al-Rashîd. Aussitôt il change d'attitude envers sa bienaimée, bien que celle-ci devienne alors plus entreprenante qu'auparavant. Il dresse même deux lits pour que chacun puisse dormir seul (p. 279). Il s'opère dans le conte un curieux renversement. A partir du moment où Ghânim décide de demeurer chaste, la jeune fille manifeste la liberté qu'elle s'est refusée jusqu'alors. Cependant Ghânim se garde de commettre l'adultère et garde durant un mois encore la fille chez lui. Il y a là, la démonstration, en un certain sens, de la fidélité pour laquelle le

${ }^{1}$ Mardrus, vol. 1, p. 276.

${ }^{2}$ Mardrus, vol. 1, p. 277. 
héros accepte une situation qui le fait souffrir. La séparation avec son amante ne viendra pas de sa part mais à cause de l'intervention du Calife lui-même.

En effet, Ghânim sera bientôt victime de la fureur du Calife qui apprend qu'il héberge sa favorite. Incapable de penser que rien ne s'est passé entre les deux, le Calife fait détruire le maison de Ghânim et cherche à s'emparer d'eux. Ghânim est contraint de fuir pour sauver sa vie et Quwat al-Qulûb est enfermée. Sur ce, selon le schéma traditionnel des amants malheureux "Ghânem, par la force de ses chagrins et les privations, tomba malade et continua à rester couché sur la vieille natte de la mosquée pendant encore un mois et il devint faible de corps et bien changé quant au teint ; et son corps fut dévoré par les puces et les punaises ; et il fut réduit à un état si misérable que les fidèles de la mosquée se concertèrent un jour entre eux pour le porter à l'hôpital de Bagdad"1. Quant à Quwat al-Qulûb, un jour Harûn alRashîd l'entend chanter une complainte dans laquelle elle loue la chasteté de Ghânim et la sévérité du Calife (p. 287). Celui-ci aussitôt se repent et délivre la favorite : "O Kouat Al-Kouloub, je t'ai entendue m'accuser d'injustice et me reprocher l'oppression : et tu as prétendu que j'avais mal agi envers celui qui m'avait fait le bien ! Qui donc est-il, celui-là qui a respecté une femme m'appartenant et dont j'ai déshonoré les femmes (sa mère et sa soeur)" ( $p$. 287).

Pour se faire pardonner à son tour son mauvais traitement, le Calife promet de donner à la favorite ce qu'elle lui demanderait. Elle réclame aussitôt Ghânim comme époux et maître "et le califat, malgré tout l'amour qu'il continuait à ressentir pour sa favorite préférée, lui dit : Cela se fera, si Allah veut ! Je te le promets d'un coeur généreux qui ne revient jamais sur ce qu'il a donné. Et il sera comblé d'honneurs !" (p. 287). Le calife tient non seulement sa promesse, puisque Ghânim épouse Quwat al-Qulûb, mais "il lui donna un palais et des émoluments somptueux et des esclaves hommes et des esclaves femmes en quantité considérable" ( p. 292). Puis le calife épouse la

${ }_{1}$ Mardrus, vol. 1, p. 286. 
soeur de Ghânim et "fit venir les scribes doués de la plus elle écriture, et leur fit écrire l'histoire de Ghânem depuis le commencement jusqu'à la fin, pour qu'elle fut conservée dans l'armoire des papiers et pût servir aux générations futures et fít l'étonnement et les délices des sages qui seraient appelés à la lire avec respect et à admirer l'oeuvre du Créateur du jour et de la nuit"(p. 292).

L'esclave de Harûn al-Rashîd devient ainsi l'épouse légitime de Ghânim élevé au plus haut rang par le Calife. L'amour a été respecté. La fidélité à l'homme aimé n'a pas été trahi et le mariage trouve dans cette illustration toute sa beauté. Cette dernière citation nous paraît avoir un sens particulier. L'histoire de Ghânim est exemplaire, c'est-à-dire la générosité du calife capable d'aller jusqu'au don de celle qu'il aime. Cette dépossession de soi est l'expression absolue du respect et de l'amour de l'autre. Il aurait pu dire : non pas comme moi je veux t'aimer mais comme toi tu désires être aimée. Le calife aime non pas l'amour comme les amants courtois mais la personne de l'autre. Il y a là une vision originale de l'amour : savoir renoncer à son sentiment pour mieux plaire à celle qu'on aime.

Décidément, les Mille et Une Nuits sont bien loin de l'adultère. Shahrayar, entendant Chéhérazade raconter de telles histoires, peut désormais reprendre confiance dans la femme. Il est donc attendu qu'il renonce définitivement à vouloir tuer les fille nubile de son royaume. Chéhérazade sera par excellence cette femme fidèle dans laquelle il peut placer toute sa confiance. 